\title{
Customization of stent design for treating malignant airway stenosis with the aid of three-dimensional printing
}

\author{
Qungang Shan ${ }^{1 \#}$, Wei Huang ${ }^{1 \#}$, Mingyi Shang ${ }^{2 \#}$, Ziyin Wang ${ }^{1}$, Ning Xia ${ }^{3}$, Qingsheng Xue ${ }^{4}$, Zhiyuan Wu ${ }^{1}$, \\ Xiaoyi Ding', Aiwu Mao', Zhongmin Wang ${ }^{1,3}$ \\ ${ }^{1}$ Department of Radiology, Ruijin Hospital, Shanghai Jiao Tong University School of Medicine, Shanghai, China; ${ }^{2}$ Department of Interventional \\ Radiology, Tongren Hospital, Shanghai Jiao Tong University School of Medicine, Shanghai, China; ${ }^{3}$ Department of Radiology, Ruijin Hospital/Lu \\ Wan Branch, Shanghai Jiao Tong University School of Medicine, Shanghai, China; ${ }^{4}$ Department of Anesthesiology, Ruijin Hospital, Shanghai Jiao \\ Tong University School of Medicine, Shanghai, China
}

"These authors contributed equally to this work.

Correspondence to: Zhongmin Wang, MD, PhD. Department of Radiology, Ruijin Hospital/Lu Wan Branch, Shanghai Jiao Tong University School of Medicine, No. 149 South Chongqing Road, Huangpu District, Shanghai 200020, China. Email: wzm0722@hotmail.com.

Background: The treatment of malignant stenosis involving the carina or bronchi is challenging due to complicated anatomy with individual variation, which makes it necessary to customize stents for each patient. Therefore, this study aims to evaluate the feasibility of a novel metallic segmented airway stent customized with the aid of three-dimensional (3D) printing for such cases.

Methods: The stents were individually customized with the aid of a 3D printed mold based on computed tomography (CT) images according to the anatomical features of the airway. A segmented design was applied on the junction part of the main stem and the branches to fit the dynamic changes of the carina angle. In 12 patients with airway stenosis caused by malignancies including esophageal cancer (EC) and lung cancer (LC), the stents were implanted. The technical and clinical success of the stenting procedure, Hugh-Jones (HJ) classification, Karnofsky performance status (KPS), and stent-related complications of patients were evaluated. Results: The stenting procedure was technically successful in all patients, and 11 patients showed significant palliation of dyspnea after stenting. The HJ and KPS classification of patients after stent insertion improved significantly compared with those before stenting ( $\mathrm{P}=0.003$ and $\mathrm{P}=0.006$, respectively). During follow-up, granulation tissue proliferation and sputum retention were found in two and four patients, respectively.

Conclusions: This study shows that the implantation of a novel stent designed with the aid of 3D printing is feasible for relieving dyspnea and improving performance status of patients with inoperable malignant stenosis involving the carina or bronchi.

Keywords: Airway; carina; malignant stenosis; stent; three-dimensional printing (3D printing)

Submitted Jun 04, 2020. Accepted for publication Oct 18, 2020.

doi: $10.21037 /$ qims-20-727

View this article at: http://dx.doi.org/10.21037/qims-20-727

\section{Introduction}

Malignant airway stenosis is a life-threatening condition caused by malignant tumors, such as locally advanced lung cancer (LC), esophageal carcinoma, and metastases, that presents with respiratory obstruction symptoms and leads to reduced quality of life $(1,2)$. Currently, airway stenting is used as an effective and minimally invasive treatment to restore airway patency in patients with inoperable malignant airway stenosis. This method can relieve airway obstruction 
symptoms immediately, thereby improving quality of life $(3,4)$.

The management of airway stenosis involving the carina or bronchi is difficult because of the complicated and irregular anatomy that is variable among different patients, although Y-shaped airway stents have been used in such cases $(5,6)$. Recently, three-dimensional (3D) printing technology has been widely used for the personalized customization of medical devices and preoperative planning (7-9). This technology has made it possible to customize patient-specific stents based on the anatomical features of the respiratory tract, thus achieving a better match between stents and airways $(5,10,11)$. Several previous studies have demonstrated the utility of $3 \mathrm{D}$ printing in the personalized customization of silicone airway stents for airway stenosis or tracheobronchomalacia $(5,12)$. However, no studies have focused on the treatment of malignant stenosis around the carina or bronchi with metallic stents customized by $3 \mathrm{D}$ printing. It should be noted that the angle between the two main bronchi may vary in patients with tumor compression or during respiration $(13,14)$. Therefore, we designed a new segmented airway stent with a changeable angle between these two branches to fit the dynamic changes of the carina angle. We have previously demonstrated the feasibility of this stent for the treatment of gastrorespiratory fistula (15). The aim of this study was to assess the feasibility of the covered metallic segmented $\mathrm{Y}$ airway stent customized with the aid of 3D printing based on computed tomography (CT) for the treatment of inoperable malignant stenosis involving the carina or bronchi.

\section{Methods}

\section{Patients}

This retrospective study was approved by our institutional review board, and written informed consent was waived. We analyzed the clinical data of patients with malignant airway stenosis involving the carina or bronchi who were treated with implantation of metallic segmented airway stent customized with the aid of 3D printing from January 2017 to May 2019. Patients with tracheoesophageal fistula or patients who underwent both airway and esophageal stenting were excluded. Finally, 12 patients were included in this study. Surgery could not be performed in these patients due to advanced tumor stages. The therapeutic strategy of airway stenting was confirmed by a multidisciplinary discussion attended by thoracic surgeons, pulmonologists, interventional radiologists, and anesthetists. The customized stent was used when the airway stenosis was anatomically complex and difficult to treat with a commercial stent. The use of the stent was approved by the institutional review board and all patients provided written informed consent prior to the procedure. Preoperative chest CT and flexible bronchoscopy were performed to help confirm the location, severity, and range of the stenosis, as well as the anatomical relationship between the airway and the surrounding structures (Figure 1A,B,C,D). The diagnosis of malignant tumors was based on the pathological biopsy results. The 7th American Joint Committee on Cancer/ Union for International Cancer Control (AJCC/UICC) staging systems were used to evaluate the tumor stage of these patients.

\section{Design of the airway stent}

All patients underwent 64-slice multidetector spiral CT (uCT760; United Imaging Healthcare, Shanghai, China) of the thorax in a supine position during end-inspiration. The scanning parameters were as follows: tube voltage, $120 \mathrm{kVp}$; tube current, $250 \mathrm{~mA}$; rotation time, $0.35 \mathrm{~s}$; pitch, 0.6; slice thickness, $1 \mathrm{~mm}$; imaging field-of-view, $320 \mathrm{~mm}$. Imaging data gathered from the chest CT scan was used to reconstruct $3 \mathrm{D}$ images of the airway using the surface rendering technique with the use of computer-assisted design software (Vitaworks, Shanghai, China). The airway and tumor were then assigned different pseudo-colors, and the image was converted into a 3D stereolithographic (STL) file (Figure 1B). Afterwards, the 3D reconstruction data was entered into a 3D printer (RS600, Union Tech, Shanghai, China) to create an airway mold that was matched 1:1 based on the anatomical characteristics of each patient's airway with photosensitive resins (Figure $2 A$ ).

The length of the stenotic segment, the diameter of the airway, the distance between the carina and the opening of the right upper lobe bronchus, and the carina angle were measured using the $3 \mathrm{D}$ printed airway mold. The measurements were performed by two trained interventional radiologists (QGS and $\mathrm{WH}$ ). Then, covered self-expandable Y-shaped metallic airway stents (MicroTech, Nanjing, China) were specifically woven with thread made of temperature-memory nickel titanium alloy with a diameter of $0.22 \mathrm{~mm}$ using the $3 \mathrm{D}$ printed airway model as a template. The diameter of the stent was $10 \%$ larger than that of the normal airway proximal to the stenosis $(16,17)$. A segmented design was applied on the junction part of the 

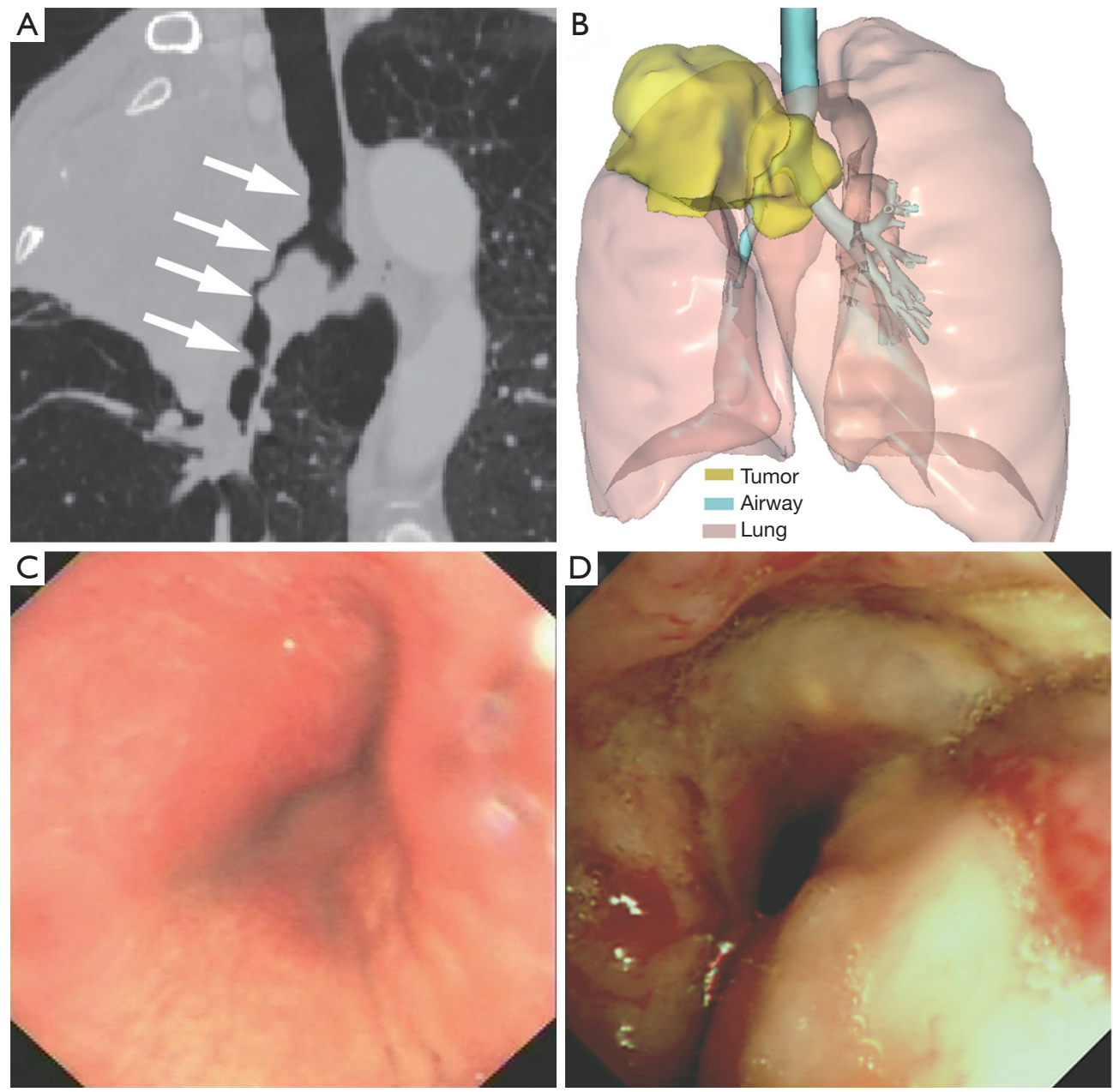

Figure 1 A 59-year-old man with airway stenosis due to the invasion of LC. (A) Oblique coronal reconstructed CT images showed the narrow airway including the lower trachea, carina, RMB, and the BI (white arrow); (B) 3D reconstruction based on CT images showed the anatomical relationship between the airway and the tumor; (C,D) bronchoscopy showed an irregular neoplasm protruding into the carina and RMB. LC, lung cancer; CT, computed tomography; RMB, right main bronchus; BI, right main bronchus; 3D, three-dimensional.

main stem and the branches using poly(tetrafluoroethylene) (PTFE) to make the stent comply with the changes in carina angle. The stent was fully coated in silicone (Figure 2B). The total manufacturing time of the stent was 3-4 days.

\section{Stent placement procedure}

The target position of the stent was determined using the $3 \mathrm{D}$ printed mold in preoperative planning. In the hybrid operation room where fluoroscopy, CT, and flexible bronchoscopy are performed in combination, an intravenous infusion of sodium lactate Ringer's solution was administered after the patient was placed in a supine position. General anesthesia was administered by oxygen inhalation with a mask. Mechanical ventilation with a 7.0 F tracheal catheter was performed by an anesthesia machine. Basic anesthesia monitoring of the blood pressure, heart rate, electrocardiogram, and oxygen saturation was continually performed for each patient during the procedure. The stent delivery system was advanced outside of the tracheal catheter under continuous oxygen supply. The location and extent of stenosis were determined under both flexible bronchoscopy and fluoroscopy guidance. The stent delivery system was introduced via 0.035 -inch stiff guidewires (Terumo, Tokyo, Japan), and then the Y stent 

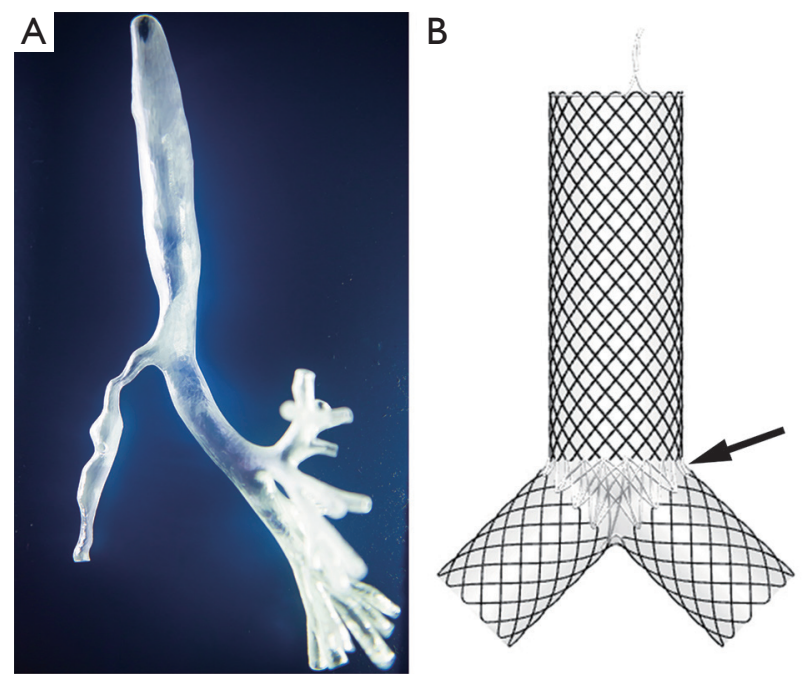

Figure 2 This is the same patient as presented in Figure 1. (A) The patient-specific airway mold was manufactured by a $3 \mathrm{D}$ printer based on 3D CT reconstruction. The severity and range of stenosis was shown clearly on the mold; (B) the segmented covered metallic Y-shaped airway stent was custom-made with the assistance of a $3 \mathrm{D}$ printed airway mold. The junction part of the main stem and the branches of the stent were made of PTFE (black arrow). 3D, three-dimensional; CT, computed tomography; PTFE, poly(tetrafluoroethylene).

was advanced and deployed across the stenosis, with its two ends located at least $1 \mathrm{~cm}$ beyond the periphery of the stenosis $(18,19)$. Immediately after stent insertion, flexible bronchoscopy and chest CT were performed to ensure the appropriate positioning and patency of the stent in the hybrid operation room without transferring the patient to a separate CT room.

\section{Follow-up}

Flexible bronchoscopy and chest CT were performed 7 days and 1 month following stent deployment to verify the patency of stents. Afterwards, the patients were followed up every 3 months if they did not show recurrent symptoms. Before and after stent deployment, the clinical symptoms were recorded, and the dyspnea grade and functional status of patients were evaluated using the Hugh-Jones (HJ) classification (20) and Karnofsky performance status (KPS) (21), respectively. Technical success was defined as proper placement of the stent at the target location across the stenotic segment with no major complications. Clinical success was defined as marked relief of dyspnea. Stent- related complications were monitored during follow-up. The aforementioned definitions were based on previous studies $(22,23)$.

\section{Statistical analysis}

The data was expressed as the median [interquartile range (IQR)]. The HJ classification and KPS before and after stenting were compared using Wilcoxon tests for paired samples. Overall median survival and cumulative survival rates were determined using the Kaplan-Meier method. SPSS 22.0 (IBM Corp., USA) and GraphPad Prism 6 (GraphPad Software Inc., USA) were used for statistical analyses. The level of statistical significance was $\mathrm{P}<0.05$ (two-tailed).

\section{Results}

\section{Patient characteristics}

The median age of the patients (10 males and 2 females) was 54 (IQR: 50.3-65) years. All patients had dyspnea, along with other clinical symptoms including cough $(\mathrm{n}=8)$ and hemoptysis $(n=1)$. Malignant stenosis was caused by LC $(n=7)$ and esophageal carcinoma $(n=5)$. Eight patients had extrinsic compression due to tumors and 4 patients had mixed airway involvement. No patients showed airway stenosis combined with fistula. All patients underwent chemotherapy and/or radiotherapy, and none of them underwent surgery before stenting. The baseline characteristics of patients are summarized in Table 1.

\section{Outcomes and follow-up}

The stenting procedures were technically successful in all 12 patients without procedure-related complications during stenting. The airway patency was restored after the stenting procedure according to bronchoscopy and CT (Figure 3). In a patient with stenosis involving the right main bronchus (RMB) and right middle bronchus, double Y-shaped stents consisting of a large and a small stent were used (Figures 4,5). A single Y-shaped stent was implanted in all the other patients. The arterial oxygen saturation values of all patients were sustained above $97 \%$ during the procedure. Bronchoscopy and chest CT after stenting showed that the stents expanded sufficiently and the airway lumen was restored immediately.

Eleven patients showed marked alleviation of their 
Table 1 Baseline characteristics of patients

\begin{tabular}{|c|c|}
\hline Characteristics & All patients $(n=12)$ \\
\hline Age $(\text { years })^{\dagger}$ & $54(50.3-65)$ \\
\hline \multicolumn{2}{|l|}{ Gender } \\
\hline Male & 10 \\
\hline Female & 2 \\
\hline \multicolumn{2}{|l|}{ Etiology } \\
\hline EC & 5 \\
\hline LC & 7 \\
\hline
\end{tabular}

Tumor stage

$\begin{array}{ll}\text { IIIA } & 3 \\ \text { IIIB } & 2 \\ \text { IIIC } & 1 \\ \text { IV } & 6\end{array}$

Previous treatment

Chemotherapy

10

Chemotherapy and radiotherapy 2

Location of stenosis

Carina, the lower trachea

LMB 3

RMB 2

Carina, RMB 3

Carina, RMB, LMB

Carina, RMB, LMB, BI 1

Carina, the lower trachea, $\mathrm{RMB}, \mathrm{BI}$

Type of stenosis

$\begin{array}{ll}\text { Extraluminal } & 8 \\ \text { Mixed } & 4\end{array}$

Tumor stage was assessed based on the 7th AJCC/UICC staging systems. ${ }^{\dagger}$, Reported as median (IQR). AJCC, American Joint Committee on Cancer; UICC, Union for International Cancer Control; IQR, interquartile range; EC, esophageal cancer; LC, lung cancer; LMB, left main bronchus; $\mathrm{RMB}$, right main bronchus; $\mathrm{BI}$, bronchus intermedius.

dyspnea after the stenting procedure. One patient with advanced esophageal cancer (EC) who had severe pneumonia did not show marked clinical improvement. The HJ classification alleviated significantly after the stenting procedure [2 (IQR: 2-3) vs. 4 (IQR: 4-5); $\mathrm{P}=0.003$ ]. The
KPS of patients after the stenting procedure improved significantly compared with that before stenting [55 (IQR: 50-60) vs. 50 (IQR: 40-50); $\mathrm{P}=0.006$ ].

The median follow-up time was 169 (IQR: 67.5-255) days. No patients showed intolerance of the stent. After stenting, all patients received tumor-specific treatment including chemotherapy and/or radiotherapy. Two patients had stent restenosis due to granulation tissue proliferation, which was treated by ablation via flexible bronchoscopy. Four patients had mucus retention, which was managed by aspiration through a bronchoscope. No patients had stent migration during follow up. Stent removal was performed in none of the patients. The median overall survival time was 181 days. The cumulative survival rates at 3 months, 6 months, and 1 year were $73.3 \%, 53.5 \%$, and $21.4 \%$, respectively. Nine patients died of tumor progression, and among these patients, 4 died within 3 months, and 3 patients were still alive at the last follow up.

\section{Discussion}

In this study, the insertion of covered metallic segmented stents customized with the aid of $3 \mathrm{D}$ printing based on CT was technically successful in all 12 patients, and 11 of the patients $(91.7 \%)$ showed significant palliation of dyspnea after stenting. This is consistent with the reported remission rate of dyspnea ranging from $70-100 \%$ in previous studies $(24,25)$. This study therefore demonstrates that the implantation of novel stents designed with the aid of $3 \mathrm{D}$ printing is feasible for treating malignant airway stenosis involving the carina or bronchi.

Malignant airway stenosis is caused by various malignant tumors, such as LC, esophageal carcinoma, and metastases. Patients with malignant stenosis present with dyspnea, cough, hemoptysis, or a combination of these symptoms $(1,2)$. A large number of patients are not able to undergo surgery due to an advanced tumor stage or poor general condition, undergoing chemotherapy and/or radiotherapy instead. For such patients, the primary aim of therapy for malignant stenosis is to reopen and maintain the airway as soon as possible to relieve respiratory symptoms, prevent obstructive pneumonia, and improve their quality of life (26-28). However, it takes some time for chemotherapy or radiotherapy to show apparent effects. In recent years, airway stenting, as a minimally invasive treatment, has become an effective method to provide immediate alleviation of dyspnea caused by malignant airway stenosis and improve quality of life $(2,4,28)$. There are various kinds 

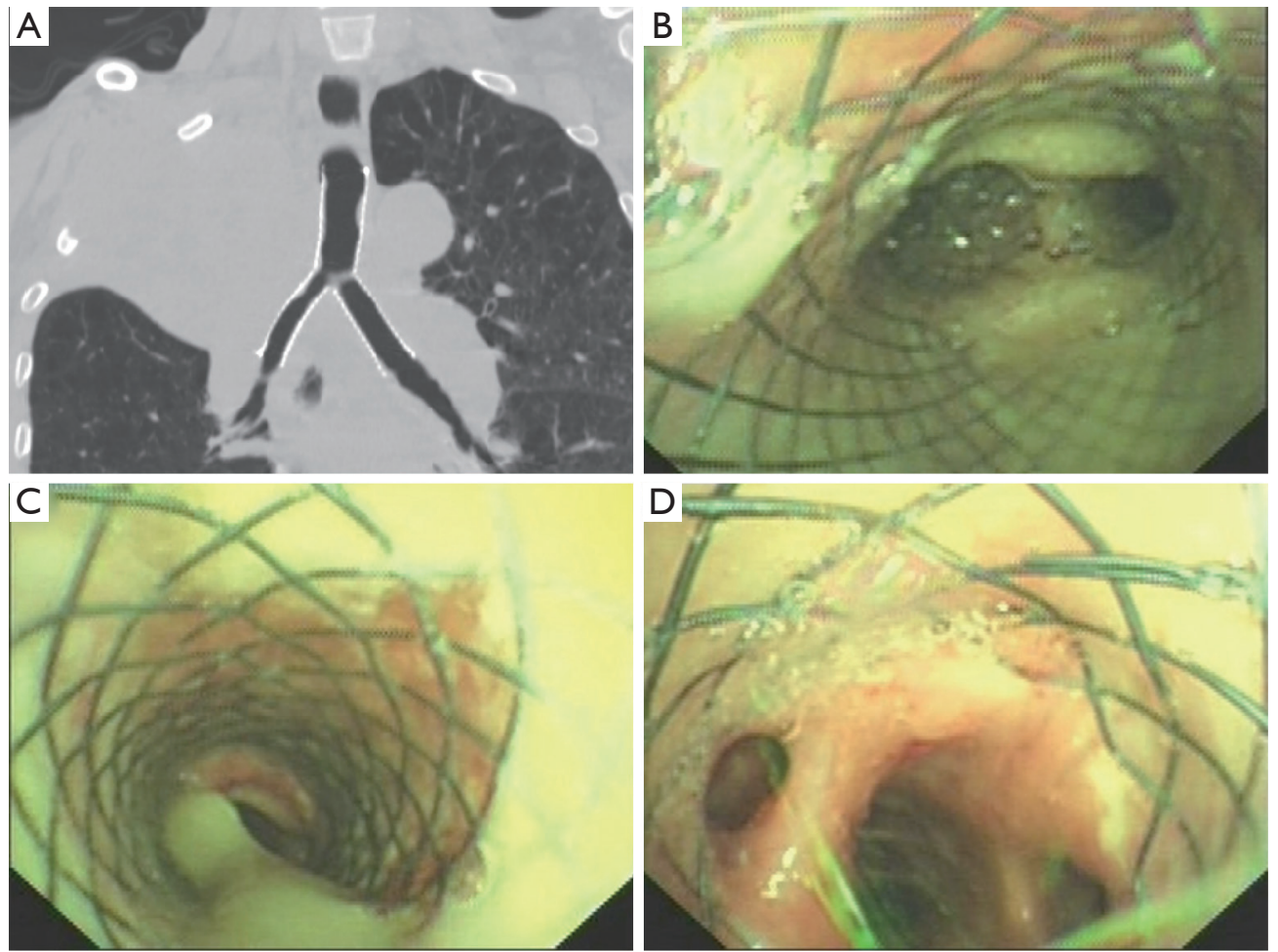

Figure 3 This is the same patient as presented in Figures 1,2. Follow-up CT and bronchoscopy 7 days after the stenting procedure. (A) Coronal reconstructed CT images showed that the airway patency was restored and the stent was in place; (B,C,D) bronchoscopy showed that the patency of the carina, RMB, and the BI was maintained by the stent. The stent was well attached to the bronchial wall. CT, computed tomography; RMB, right main bronchus; BI, right main bronchus.
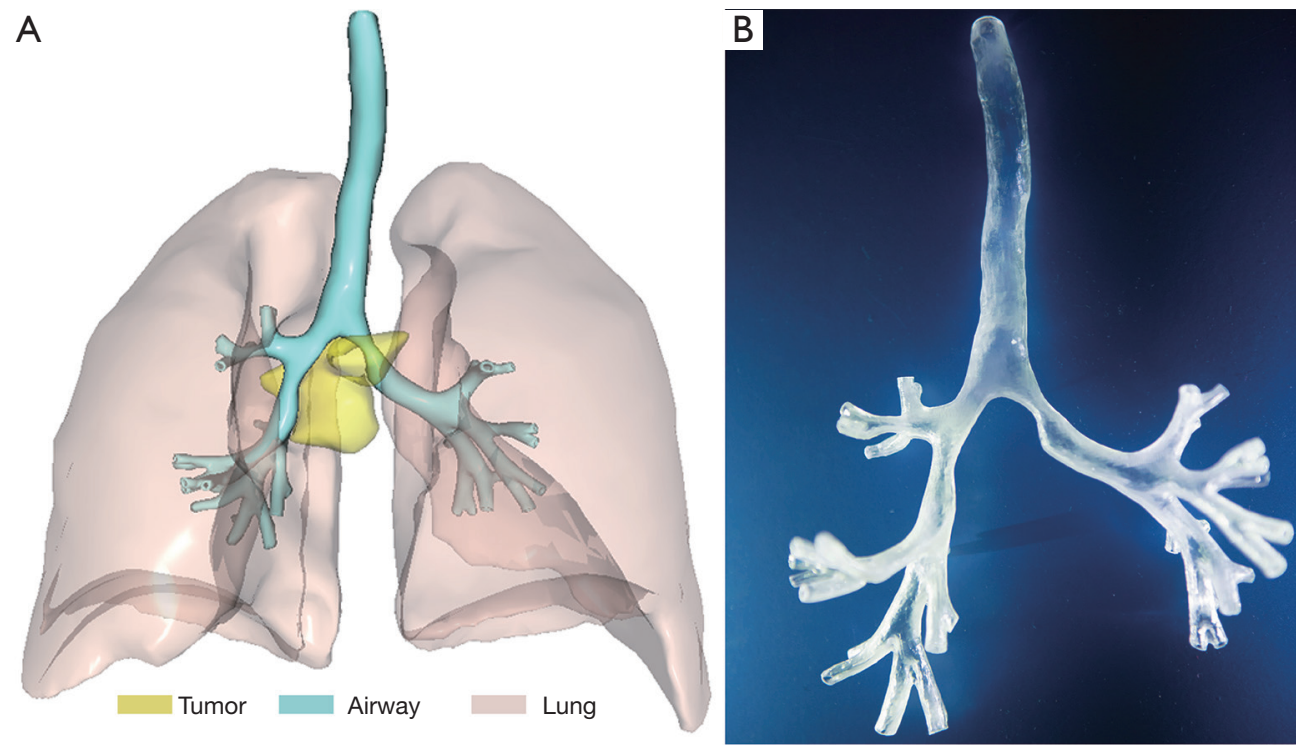

Figure 4 A 71-year-old woman with airway stenosis due to invasion of EC. (A) 3D CT reconstruction image showed that the stenotic region of airway was compressed by the tumor. The narrow airway included the carina, LMB, RMB, and the BI; (B) the patient-specific airway mold was manufactured by a 3D printer based on 3D CT reconstruction. EC, esophageal cancer; 3D, three-dimensional; CT, computed tomography; LMB, left main bronchus; RMB, right main bronchus; BI, right main bronchus. 

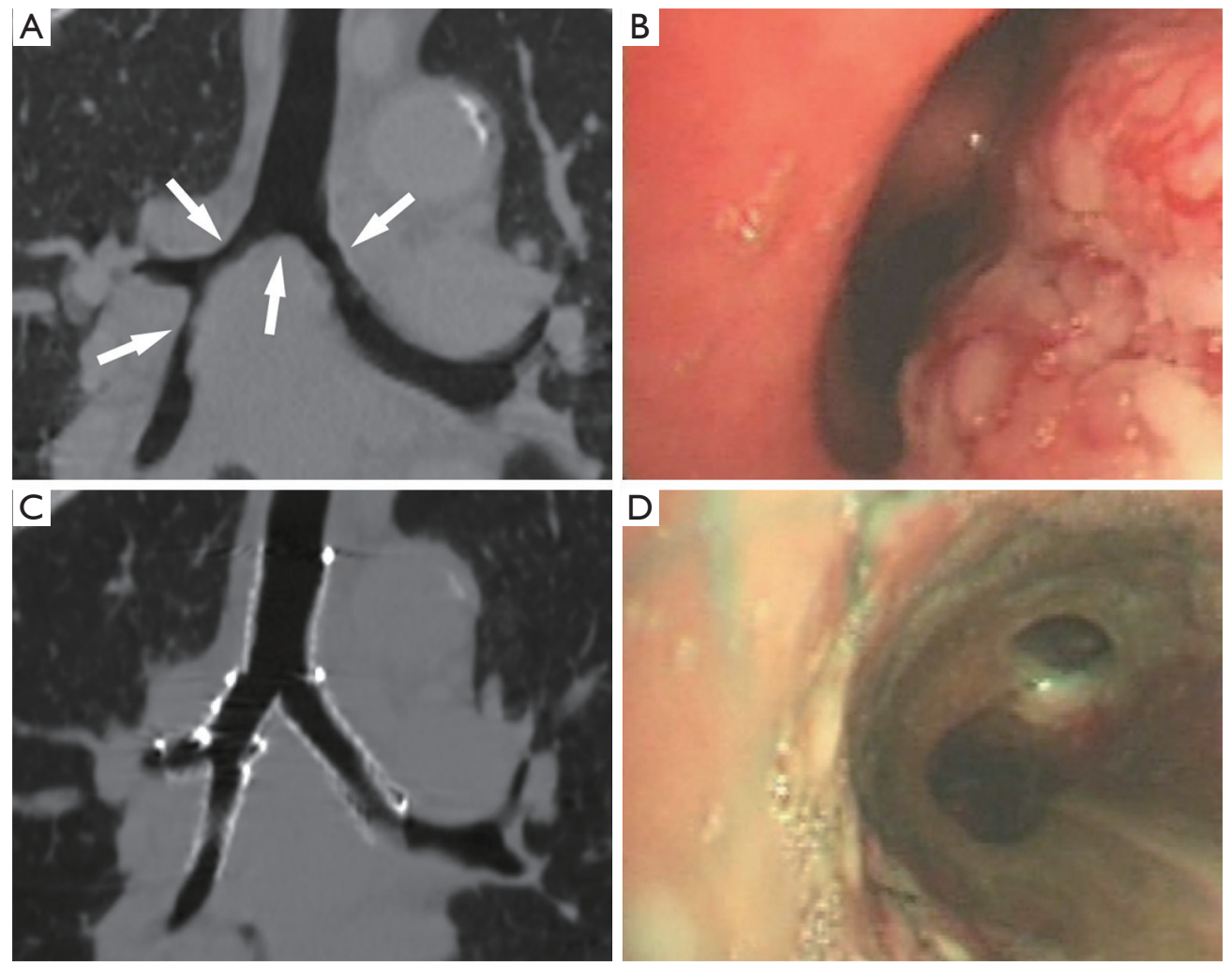

Figure 5 CT and bronchoscopy images of the patient before and after double Y stenting. (A) Coronal reconstructed CT images showed the narrow airway including the carina, LMB, RMB, and the BI (white arrow); (B) bronchoscopy showed an irregular neoplasm protruding into the RMB; (C) coronal reconstructed CT images showed that the airway patency was restored and the 2 Y-shaped stents were in place; (D) bronchoscopy showed that the RMB was patent. CT, computed tomography; LMB, left main bronchus; RMB, right main bronchus; BI, right main bronchus.

of stents using different materials that have their respective advantages and disadvantages $(24,29,30)$. Covered metallic stents have been widely used in malignant stenosis and can decrease the rate of granulation tissue proliferation and tumor ingrowth compared with uncovered metallic stents $(29,30)$. In this study, we focused on malignant airway stenosis involving the carina or bronchi. Therefore, covered metallic Y-shaped airway stents were used. In some cases, extensive airway stenosis or fistula involving the trachea and bronchus can not be covered completely by a single $\mathrm{Y}$ stent. Previous studies have reported the feasibility of double Y-shaped stenting for such cases $(31,32)$. In this study, double $Y$ stenting was performed in one patient. The stenotic region was fully covered by the combination of two $\mathrm{Y}$ stents without covering the opening of the right upper lobe bronchus. Nevertheless, the efficacy and safety of double $Y$ stenting requires further studies.

The treatment of anatomically complicated stenosis involving the carina or bronchi is challenging. The compatibility of commercially available airway stents is not satisfactory, and thus may lead to poor effectiveness and complications $(5,10)$. Therefore, it is necessary to customize personalized stents to fit the anatomy of an individual patient's airway. With the aid of 3D printing and computerassisted design, airway stents can be customized according to the dimensions of the airway, which vary greatly among different patients, especially in the anatomically complex carina region. Personalized customized stents therefore provide a better match to the airway, thus improving outcomes and reducing the risk of complications. Several previous studies have reported the application of patientspecific silicone stents manufactured by silicone injections into $3 \mathrm{D}$ printed airway molds, and promising outcomes were obtained $(5,10,12)$. However, no previous study has reported the use of metallic stents customized with the assistance of 3D printing, except for our previous studies 
that reported the use of such stents for gastrorespiratory fistula $(15,33)$. In this study, we evaluated the feasibility of metallic stents customized with the assistance of $3 \mathrm{D}$ printing for patients with malignant stenosis. The stents achieved a good match to the airway dimensions, and airway patency was effectively restored. In addition, the application of a $3 \mathrm{D}$ printed mold strengthened the doctor's comprehensive understanding of individual patient anatomical features, and helped doctors plan the accurate location of stenting to cover the stenotic segment before the stenting procedure. However, it should be noted that the manufacturing of such stents requires several days, and would therefore not be applicable for patients in an emergency setting. The added value of 3D printing in airway stenting for anatomically complex airway stenosis requires further validation by future studies.

The angle of the carina in the airway is not static, and it changes $10^{\circ}$ or more with breathing movements and different postures in a normal person (14). In particular, for patients with malignant tumors involving the airway, a $20^{\circ}-30^{\circ}$ change in the angle of the carina should be considered due to the compression on the airway caused by the malignancy (13). In addition, for such patients, stent migration may occur due to tumor shrinkage after tumorspecific therapy (34). To match the dynamic changes in the carina angle, we designed a novel segmented stent. The junction of the three separated parts of the stent was made of PTFE—a soft material, which enables the stent to adapt to changes in the carina angle. If the carina angle varies with the shrinkage or enlargement of the tumor after stenting, the segmented stent may adapt to the change better than a conventional wholly knitted stent, and thus the irritation of the stent on the bronchial wall, which may cause intolerance to the stent, is reduced. In the current study, the stents fitted well with the airways, and no intolerance of the stents or stent migration was found during follow up. Further studies will be required to assess the clinical benefits of the segmented stent. In addition, the flexible joints of the novel stents may make the precision of the angle of the stent less necessary compared with conventional stents. We have carried out a biomechanical experiment to test how the pressure of the stent on the bronchial wall changes with the angle of stent, and the results may provide guidance for stent customization. In clinical practice, we still try to make the angle of the stent consistent with that of the airway to reduce the stimulation on the bronchial wall in nonemergency cases.

Airway stenting can not only relieve the symptoms caused by airway obstruction as a palliative therapy, but also can act as a bridging therapy to enable additional tumor-specific treatments, including radiotherapy or chemotherapy, after stenting $(26,35)$. In patients with a good response after airway stenting and subsequent tumor-specific therapy that provides survival benefits and durable relief of dyspnea by reducing tumor burden, stent removal may be considered (36). In this study, although tumor reduction was achieved to a different extent in some patients after tumor-specific therapy, stent removal was not performed because of the advanced tumor stage and potential risks of stent removal.

In this study, general anesthesia was administered, and constant ventilation was provided through a smalldiameter tracheal tube, and the paths for oxygen supply and the stenting procedure were separated. Consequently, this provided enough time for the stenting procedure and therefore increased the accuracy of positioning and reduced procedural-related complications. In the current study, the stents were deployed accurately, and no major complications occurred during the procedure. In addition, the relative ease of the stenting procedure carries the potential to reduce the over-reliance on personal experience, and promotes popularization of the procedure.

There were several limitations in this study. First, there was no control group for comparison in this study. Second, the sample size was small. Third, the etiologies of the malignant stenoses were heterogeneous. Fourth, due to the advanced tumor stage of the included patients in this study, the survival time of patients was limited. Therefore, there was a lack of mid and long-term follow-up data of the stenting procedure. Further studies with a larger sample size and control group should therefore be performed to validate our conclusions. In addition, the inclusion of more patients who are sensitive to tumor-specific treatment will enable mid and long-term follow-up of the stenting procedure.

In conclusion, this proof-of-concept study illustrated that implantation of novel covered metallic segmented $\mathrm{Y}$ airway stents customized with $3 \mathrm{D}$ printing based on CT was feasible for relieving dyspnea and improving the performance status of patients with inoperable malignant stenosis involving the carina or bronchi. The long-term outcomes of this stent, designed with the aid of 3D printing, requires further study.

\section{Acknowledgments}

Funding: Supported by the Shanghai Key Specialty Construction Project (No. ZK2019A02); Clinical Key Specialist Construction Project of Shanghai Municipal 
Health Commission [Interventional Radiology (No. shslczdzk06002) \& 3D Printing (No. shslczdzk07002)]; Shanghai Municipal Commission of Health and Family Planning (No. 201640087).

\section{Footnote}

Conflicts of Interest: All authors have completed the ICMJE uniform disclosure form (available at http://dx.doi. org/10.21037/qims-20-727). Dr. ZW reports grants from Shanghai key specialty construction project, grants from Clinical key specialist construction project of Shanghai municipal health commission during the conduct of the study. In addition, Dr. ZW has a patent A segmented bifurcated stent issued. Dr. WH reports grants from Shanghai municipal commission of health and family planning during the conduct of the study. The other authors have no conflicts of interest to declare.

Ethical Statement: This retrospective study was approved by our institutional review board and written informed consent was waived.

Open Access Statement: This is an Open Access article distributed in accordance with the Creative Commons Attribution-NonCommercial-NoDerivs 4.0 International License (CC BY-NC-ND 4.0), which permits the noncommercial replication and distribution of the article with the strict proviso that no changes or edits are made and the original work is properly cited (including links to both the formal publication through the relevant DOI and the license). See: https://creativecommons.org/licenses/by-nc-nd/4.0/.

\section{References}

1. Phillips MJ. Malignant airway obstruction: what to do? Respirology 2018;23:803-4.

2. Murgu SD, Egressy K, Laxmanan B, Doblare G, OrtizComino R, Hogarth DK. Central airway obstruction: benign strictures, tracheobronchomalacia, and malignancyrelated obstruction. Chest 2016;150:426-41.

3. Ong P, Grosu HB, Debiane L, Casal RF, Eapen GA, Jimenez CA, Noor L, Ost DE. Long-term quality-adjusted survival following therapeutic bronchoscopy for malignant central airway obstruction. Thorax 2019;74:141-56.

4. Huang S, Xu J, An Z, Yuan P, Xu H, Lv W, Hu J. Clinical assessment of airway stent placement in patients with malignant airway lesions. J Thorac Dis 2018;10:3277-88.
5. Guibert N, Didier A, Moreno B, Lepage B, Leyx P, Plat G, Mhanna L, Murris M, Mazières J, Hermant C. Treatment of complex airway stenoses using patient-specific 3D-engineered stents: a proof-of-concept study. Thorax 2019;74:810-3.

6. Xu J, Ong HX, Traini D, Byrom M, Williamson J, Young PM. The utility of 3D-printed airway stents to improve treatment strategies for central airway obstructions. Drug Dev Ind Pharm 2019;45:1-10.

7. Sun Z, Jansen S. Personalized $3 \mathrm{D}$ printed coronary models in coronary stenting. Quant Imaging Med Surg 2019;9:1356-67.

8. Allan A, Kealley C, Squelch A, Wong YH, Yeong CH, Sun Z. Patient-specific 3D printed model of biliary ducts with congenital cyst. Quant Imaging Med Surg 2019;9:86-93.

9. Hoetzenecker K, Chan HHL, Frommlet F, Schweiger T, Keshavjee S, Waddell TK, Klepetko W, Irish JC, Yasufuku $\mathrm{K} .3 \mathrm{D}$ models in the diagnosis of subglottic airway stenosis. Ann Thorac Surg 2019;107:1860-5.

10. Gildea TR, Young BP, Machuzak MS. Application of 3D printing for patient-specific silicone stents: 1 -year followup on 2 patients. Respiration 2018;96:488-94.

11. Cheng GZ, Folch E, Brik R, Gangadharan S, Mallur P, Wilson JH, Husta B, Majid A. Three-dimensional modeled T-tube design and insertion in a patient with tracheal dehiscence. Chest 2015;148:e106-8.

12. Schweiger T, Gildea TR, Prosch H, Lang G, Klepetko W, Hoetzenecker K. Patient-specific, 3-dimensionally engineered silicone Y-stents in tracheobronchomalacia: clinical experience with a novel type of airway stent. J Thorac Cardiovasc Surg 2018;156:2019-21.

13. Freitag L, Eicker R, Linz B, Greschuchna D. Theoretical and experimental basis for the development of a dynamic airway stent. Eur Respir J 1994;7:2038-45.

14. Chen JT, Putman CE, Hedlund LW, Dahmash NS, Roberts L. Widening of the subcarinal angle by pericardial effusion. AJR Am J Roentgenol 1982;139:883-7.

15. Huang W, Shan Q, Wu Z, Li H, Zhou M, Ding X, Wang Z. Retrievable covered metallic segmented Y airway stent for gastrorespiratory fistula of carina or main bronchi. J Thorac Cardiovasc Surg 2020. [Epub ahead of print]. doi: 10.1016/j.jtcvs.2020.03.019.

16. Charokopos N, Foroulis CN, Rouska E, Sileli MN, Papadopoulos N, Papakonstantinou C. The management of post-intubation tracheal stenoses with self-expandable stents: early and long-term results in 11 cases. Eur J Cardiothorac Surg 2011;40:919-24.

17. Han X, Al-Tariq Q, Zhao Y, Li L, Cheng Z, Wang H, Liu 
C, Jiao D, Wu G. Customized hinged covered metallic stents for the treatment of benign main bronchial stenosis. Ann Thorac Surg 2017;104:420-5.

18. Ma J, Han X, Wu G, Jiao D, Ren K, Bi Y. Outcomes of temporary partially covered stent placement for benign tracheobronchial stenosis. Cardiovasc Intervent Radiol 2016;39:1144-51.

19. Kim MJ, Shin JH, Park JH, Kim JH, Song HY, Park J, Cho YC, Kim SH. Covered airway stent placement for malignant tracheobronchial strictures in patients with an endotracheal tube. Clin Radiol 2016;71:1120-5.

20. Nakahara Y, Yasunaga H, Inokuchi H, Ogata N, Horiguchi H, Matsuda S, Fushimi K, Haga N. Mortalityreducing effect of rehabilitation for COPD: observational propensity-matched cohort study using a Nationwide Database. Respir Care 2016;61:1497-504.

21. Fraser SD, Barker J, Roderick PJ, Yuen HM, Shardlow A, Morris JE, McIntyre NJ, Fluck RJ, McIntyre CW, Taal MW. Health-related quality of life, functional impairment and comorbidity in people with mild-to-moderate chronic kidney disease: a cross-sectional study. BMJ Open 2020;10:e040286.

22. Kim J, Shin JH, Kim J, Song H, Song S, Park CK. Metallic stent placement for the management of tracheal carina strictures and fistulas: technical and clinical outcomes. AJR Am J Roentgenol 2014;202:880-5.

23. Shin B, Chang B, Kim H, Jeong B. Interventional bronchoscopy in malignant central airway obstruction by extra-pulmonary malignancy. BMC Pulm Med 2018;18:46.

24. Herth FJF, Eberhardt R. Airway stent: what is new and what should be discarded. Curr Opin Pulm Med 2016;22:252-6.

25. Furukawa K, Ishida J, Yamaguchi G, Usuda J, Tsutsui H, Saito M, Konaka C, Kato H. The role of airway stent placement in the management of tracheobronchial stenosis caused by inoperable advanced lung cancer. Surg Today 2010;40:315-20.

26. Ost DE, Ernst A, Grosu HB, Lei X, Diaz-Mendoza J, Slade M, Gildea TR, Machuzak MS, Jimenez CA, Toth J, Kovitz KL, Ray C, Greenhill S, Casal RF, Almeida FA, Wahidi MM, Eapen GA, Feller-Kopman D, Morice
RC, Benzaquen S, Tremblay A, Simoff M, AQuIRE BR. Therapeutic bronchoscopy for malignant central airway obstruction: success rates and impact on dyspnea and quality of life. Chest 2015;147:1282-98.

27. Gompelmann D, Eberhardt R, Herth FJF. Advanced malignant lung disease: what the specialist can offer. Respiration 2011;82:111-23.

28. Simoff MJ, Lally B, Slade MG, Goldberg WG, Lee P, Michaud GC, Wahidi MM, Chawla M. Symptom management in patients with lung cancer: Diagnosis and management of lung cancer, 3rd ed: American College of Chest Physicians evidence-based clinical practice guidelines. Chest 2013;143:e455S-e497S.

29. Ayub A, Al-Ayoubi AM, Bhora FY. Stents for airway strictures: selection and results. J Thorac Dis 2017;9:S116-21.

30. Avasarala SK, Freitag L, Mehta AC. Metallic endobronchial stents. Chest 2019;155:1246-59.

31. Oki M, Saka H. Double Y-stenting for tracheobronchial stenosis. Eur Respir J 2012;40:1483-8.

32. Li TF, Duan XH, Han XW, Wu G, Ren JZ, Ren KW, Lu HB. Application of combined-type Y-shaped covered metallic stents for the treatment of gastrotracheal fistulas and gastrobronchial fistulas. J Thorac Cardiovasc Surg 2016;152:557-63.

33. Han Y, Yang S, Huang W, Wang Z, Li H. A Hem-o-Lokinduced tracheoesophageal fistula cured by temporary airway stenting modified with three-dimensional printing. Ann Thorac Surg 2018;106:e219-21.

34. Godoy MC, Saldana DA, Rao PP, Vlahos I, Naidich DP, Benveniste MF, Erasmus JJ, Marom EM, Ost D. Multidetector CT evaluation of airway stents: what the radiologist should know. Radiographics 2014;34:1793-806.

35. Mallow C, Thiboutot J, Semaan R, Hayes MM, Hales R, Ram A, Feller-Kopman D, Lee H, Yarmus L. External beam radiation therapy combined with airway stenting leads to better survival in patients with malignant airway obstruction. Respirology 2018;23:860-5.

36. Oki M, Saka H, Hori K. Airway stenting in patients requiring intubation due to malignant airway stenosis: a 10-year experience. J Thorac Dis 2017;9:3154-60.
Cite this article as: Shan Q, Huang W, Shang M, Wang Z, Xia N, Xue Q, Wu Z, Ding X, Mao A, Wang Z. Customization of stent design for treating malignant airway stenosis with the aid of three-dimensional printing. Quant Imaging Med Surg 2021;11(4):1437-1446. doi: 10.21037/qims-20-727 